\title{
Imaging Evaluation of Mesenteric Ischemia: Is There a Golden Period for This Entity?
}

\author{
Ishfaq Zargar ${ }^{1}$, Irfan Robanni ${ }^{1}$, Omair Shah ${ }^{1 *}$ (D), Tariq Gojwari, Riaz Rasool ${ }^{1}$, \\ Naseer Choh ${ }^{1}$, Faiz Shera ${ }^{1}$, Munir Wani ${ }^{2}$
}

\begin{abstract}
Background. The study was aimed at assessing the role of ultrasonography and multidetector computed tomography angiography in evaluating patients with suspected mesenteric ischemia, as well as assessing the effect of the time from presentation to management on mortality and morbidity.

Materials and Methods. Patients with clinically suspected mesenteric ischemia underwent Doppler ultrasound and contrast-enhanced computed tomography. On ultrasonography, we assessed any filling defect in the superior mesenteric artery/vein, narrowing or occlusion of the proximal superior mesenteric artery, ascites, bowel wall thickening, and pneumatosis/portal venous gas. Computed tomography angiography was performed looking for any filling defect in the superior mesenteric artery/vein, superior mesenteric artery/vein calibre, bowel wall thickening, calibre and enhancement and pneumatosis/portal vein gas. Most of our patients underwent emergency surgery and the findings correlated with imaging. All the patients were divided into Group A $(n=30)$ and Group B $(n=17)$ based on the time from presentation to management: within 48 hours of presentation and 48 hours after presentation, respectively.

Results. On computed tomography scan, mesenteric vascular involvement was seen in 27 (55\%) patients, mesenteric/intestinal twist was observed in 12 (25\%) patients, and non-occlusive mesenteric ischemia was found in $6 \%$ of patients. The computed tomography findings were found to have a sensitivity of $86 \%$, a specificity of $94 \%$ and an accuracy of $90 \%$ in cases of mesenteric ischemia. Among 35 patients operated on, those presenting within 48 hours, had a significantly less mortality $(63 \%)$ in comparison to those presenting after 48 hours (90\%).

Conclusions. Clinical, laboratory and ultrasound features are non-specific in diagnosing mesenteric ischemia. Computed tomography angiography is a sine qua non in mesenteric ischemia diagnosis. Patients with venous ischemia respond well to conservative management. Early intervention within the first 48 hours is associated with better prognosis.
\end{abstract}

\section{Keywords}

Computed Tomography Angiography; Mesenteric Ischemia; Ultrasonography; Non-Occlusive Mesenteric Ischemia

${ }^{1}$ Department of Radiology, Sher-I-Kashmir Institute of Medical Sciences, Soura, Srinagar, Jammu and Kashmir, India ${ }^{2}$ Department of General and Minimal Invasive Surgery, Sher-I-Kashmir Institute of Medical Sciences, Soura, Srinagar, Jammu and Kashmir, India

${ }^{*}$ Corresponding author: shahomair133@gmail.com

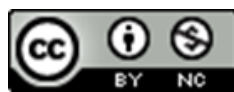

Copyright @Ishfaq Zargar, Irfan Robanni, Omair Shah, Tariq Gojwari, Riaz Rasool, Naseer Choh, Faiz Shera, Munir Wani 2022

\section{Introduction}

Mesenteric ischemia is characterized by insufficient blood supply to the intestine, resulting from vascular obstruction, vascular or intestinal twist or a generalized hypoperfusion state. It can be acute (sudden severe abdominal pain; is associated with a high risk of death) or chronic (gradual, with post-prandial pain, fear of eating, vomiting, weight loss) [1]. Mortality rates from mesenteric ischemia are high, ranging from $30 \%$ to $90 \%$ even after surgical in- tervention [2]. Those undergoing surgery usually have a poor functional outcome in view of the short residual bowel, often leading to malabsorption and malnutrition [3]. A diagnosis of mesenteric ischemia is essential, although difficult, to give the patient some chance of overcoming this dangerous condition. A high index of suspicion is required from both the clinician and the radiologist. Familiarity with the imaging spectrum associated with mesenteric ischemia is required to ensure prompt recognition of the disease. 
Clinical and laboratory findings (serum lactate) can sometimes point to the diagnosis of mesenteric ischemia, but can be misleading $[4,5]$. The various imaging modalities used for mesenteric ischemia include an abdominal radiograph, ultrasonography (USG), computed tomography angiography (CT angiography), magnetic resonance angiography and conventional angiography. A radiograph is quite non-specific; however, it may show the presence of air-fluid levels, free gas under diaphragm and portal venous (PV) gas in some cases. Doppler USG has recently been studied in cases of mesenteric ischemia, showing the presence of bowel wall thickening, bowel dilatation, lack of peristalsis and ascites [6]. The imaging modality of choice presently is CT angiography with a pooled sensitivity of $93.3 \%$ and a pooled specificity of $95.9 \%$ for diagnosing acute mesenteric ischemia [7]. CT has the advantage of showing not only the mesenteric vasculature, directly detecting any filling defects, but also structural causes of ischemia, including closed loop hernias, internal hernias, and features of non-occlusive mesenteric ischemia (NOMI). Although angiography is accurate in diagnosing vascular occlusions, its use is nowadays limited to conditions when endovascular intervention is planned. Magnetic resonance angiography can be used with the advantage of no radiation, but it is a time-consuming study, with availability being a major limiting factor. In addition, peristalsis can cause major artifacts and vascular assessment does not correspond to the level achieved by conventional or CT angiography. CT angiography, therefore, remains the investigation to go to in all patients with suspected mesenteric ischemia [8]. As the prognosis is poor, treatment should start promptly. An emergent laparotomy is the usual treatment in cases with peritonitis and signs of perforation [9]. In patients without frank evidence of bowel infarction, endovascular treatment may be helpful in restoring blood flow.

Venous ischemia, on the other hand, is somewhat benign and usually requires non-surgical treatment with anticoagulation (heparin followed by oral anticoagulants). Management of NOMI is based mainly on correcting the underlying cause of reduced intestinal perfusion [10]. Intravascular infusion of vasodilators (e.g., papaverine hydrochloride) may be advantageous in some cases $[11,12]$.

Based on the difficulty of diagnosing this condition owing to the varied presentation and associated high mortality and morbidity, our study aimed to evaluate specific imaging features that could guide management. We also aimed to ascertain the effect of early CT angiography on early diagnosis and management.

\section{Materials and Methods}

\section{Study Design}

Our study was a prospective observational study conducted in the Department of Radio Diagnosis and Imaging, Sher-IKashmir Institute of Medical Sciences (SKIMS), including patients referred by the Department of General Surgery from 2017 to 2020. Demographic profile of all the patients was noted. All our patients were initially evaluated for any predisposing factors and clinically examined for signs of peritonitis. The time from first presentation to the imaging documentation was noted in all cases. Baseline investigations were done, and serum lactate levels were obtained in all the cases.

\section{Study Population}

All patients suspected of having mesenteric ischemia presenting to the emergency medicine department were included in the study. Patients with contrast allergy, children below 18 years of age, pregnant and lactating females were excluded from the study.

\section{Imaging Protocol}

The imaging protocol at our institution included an USG followed by immediate CT angiography in all patients. Doppler USG was performed by a radiologist with an experience of 4 years, using the GE Logiq P5 ultrasound machine. Various parameters assessed by USG included any filling defect in the superior mesenteric artery (SMA) /superior mesenteric vein (SMV), presence of ascites, bowel wall thickening and pneumatosis/PV gas.

All patients were examined with a multi-detector row CT scanner (CT 64 SOMATOM) using $1.5-2 \mathrm{ml} / \mathrm{kg}$ of nonionic intravenous contrast agent (Omnipaque/Contrapaque $300 \mathrm{mg} / \mathrm{ml}$ ) through an 18-gauge antecubital intravenous line at a rate of $4 \mathrm{~mL} / \mathrm{sec}$. Before contrast administration, a non-contrast scan of the abdomen was obtained in all the patients. Bolus tracking technique was used, keeping the trigger on the proximal abdominal aorta with a threshold of 120-130 HU and a scan delay of 8 seconds; scanning was performed with $1.25-\mathrm{mm}$ collimation, $7.50-\mathrm{mm}$ pitch and high-speed mode. Images in this phase were obtained from above the level of the celiac axis to below the level of the aortic bifurcation. Thereafter, a conventional portal phase acquisition was obtained at 60-70 seconds after injection with 5-mm collimation, 11.25-mm pitch and highquality mode from above the level of the diaphragm to below the level of the symphysis pubis. Images were transferred to a dedicated workstation and reconstructed into contiguous $1.5-\mathrm{mm}$ transverse images in the angiographic phase and PV phase. The parameters assessed by CT included the presence of filling defect in the SMA/SMV, SMA/SMV calibre, bowel wall thickening, calibre, and enhancement. Bowel wall thickness of greater than $3 \mathrm{~mm}$ in the small bowel and $5 \mathrm{~mm}$ in the large bowel was considered thickened. Small bowel dilatation was considered when the maximum diameter exceeded $3 \mathrm{~cm}$ and large bowel dilatation was considered when the maximum diameter exceeded $5 \mathrm{~cm}$. Other parameters assessed by CT were the presence of ascites and pneumatosis/PV gas.

Many of our patients underwent an emergent laparotomy based on clinical, laboratory and imaging findings. The surgical findings were correlated with the CT findings, mainly focussing on the important question of the presence or absence of bowel ischemia and the extent of the disease.

\section{Statistical Analysis}

The data were collected, and statistical analysis was done using SPSS 22.0; categorical data are presented as per- 
Table 1. Ultrasound findings of patients with different etiology of mesenteric ischemia.

\begin{tabular}{|c|c|c|c|c|c|c|c|}
\hline Etiology & $\begin{array}{l}\text { Arterial } \\
\text { thrombus }\end{array}$ & $\begin{array}{l}\text { Venous } \\
\text { thrombus }\end{array}$ & $\begin{array}{c}\text { Bowel } \\
\text { thickening }\end{array}$ & $\begin{array}{c}\text { Bowel } \\
\text { distention }\end{array}$ & Ascites & $\begin{array}{c}\text { Portal } \\
\text { venous gas }\end{array}$ & Pneumatosis \\
\hline Arterial occlusion $(n=19)$ & $5(26 \%)$ & $2(10.5 \%)$ & $8(42 \%)$ & $7(37 \%)$ & $13(63 \%)$ & $2(10.5 \%)$ & $3(16 \%)$ \\
\hline Venous occlusion $(n=13)$ & $2(15 \%)$ & $6(46 \%)$ & $9(69 \%)$ & $4(31 \%)$ & $10(77 \%)$ & NONE & $1(7.6 \%)$ \\
\hline Mesenteric/intestinal twist $(n=12)$ & NONE & NONE & $1(8 \%)$ & $12(100 \%)$ & $8(67 \%)$ & $1(8 \%)$ & NONE \\
\hline $\operatorname{NOMI}(n=3)$ & NONE & NONE & $3(100 \%)$ & $2(67 \%)$ & $3(100 \%)$ & NONE & $1(33 \%)$ \\
\hline
\end{tabular}

centages; continuous variables are presented as means and standard deviations.

\section{Results}

\section{Demographic Profile}

Our study included a total of 47 patients having a mean age of $57 \pm 11$ years (range: 40 to 75 years) with a male-tofemale ratio of $30(64 \%)$ : $17(36 \%)$. Most of our patients were managed in the peripheral hospitals for some time before presenting to our tertiary care center.

\section{Clinical Profile}

The major presenting feature in our patients was abdominal pain $(n=45,96 \%)$, nausea/vomiting $(n=30,64 \%)$, gastrointestinal bleeding $(\mathrm{n}=19,40 \%)$ and signs of peritonitis $(n=10,21 \%)$. Based on these symptoms, all our patients had a suspicion of mesenteric ischemia.

We observed the time from first presentation to imaging in all our patients as well. The average time of presentation was $40 \pm 15$ hours. We divided our patients into two groups based on the time of imaging: Group A with imaging and treatment done 48 hours after presentation and group B with imaging and management within 48 hours of presentation. We had $30(64 \%)$ patients in Group A and $17(36 \%)$ patients in Group B. This grouping was done based on a retrospective assessment of mortality among our patients as it was obvious that those presenting late ( $>48$ hours) had increased mortality.

\section{Serum Lactate}

Among the biochemical markers, serum lactate increased $(>2 \mathrm{mmol} / \mathrm{L})$ in $20(42 \%)$ patients, while it was normal $(<2 \mathrm{mmol} / \mathrm{L})$ in $27(58 \%)$ patients at the time of presentation.

\section{Cause of Ischemia}

Mesenteric arterial thrombus/embolus was seen in 19 (40\%) patients, while mesenteric venous occlusion was the cause in $13(28 \%)$ patients. We had $12(25 \%)$ patients with ischemia secondary to mesenteric or intestinal twisting, while the remaining $3(7 \%)$ patients had NOMI.

\section{Imaging Findings Ultrasonography}

The most common finding on USG was ascites seen in $34(72 \%)$ patients followed by bowel distention seen in $25(53 \%)$ patients. The other findings included venous thrombosis $(\mathrm{n}=8,17 \%)$, arterial thrombosis $(\mathrm{n}=5,10 \%)$, bowel wall thickening $(n=20,42 \%)$ and pneumatosis intestinalis/PV gas $(\mathrm{n}=8,17 \%)$. USG identified a volvulus in $3(6 \%)$ patients (Table 1$)$.

\section{Contrast Enhanced Computed Tomography}

As in case of ultrasonography, the most common finding on CT was ascites seen in $39(83 \%)$ patients followed by bowel thickening in $31(66 \%)$ patients. Arterial and venous thromboses were seen in $17(36 \%)$ and $12(26 \%)$ patients, respectively. Bowel distention was noted in $33(70 \%)$ patients; pneumatosis intestinalis was observed in 23 (49\%) patients; PV gas was found in $9(19 \%)$ patients. Other findings on CT included mesenteric congestion $(n=29,62 \%)$, volvulus $(n=3,6 \%)$ and closed loop obstruction $(n=10$, $21 \%$ ). Bowel enhancement was assessed in the PV phase, and we found decreased enhancement in $26(55 \%)$ patients, while normal/increased bowel enhancement was seen in 21 $(45 \%)$ patients. Free gas in the peritoneum was rare and seen in $3(6 \%)$ patients (Table 2$)$.

\section{Correlation Between Surgical and Imaging Findings}

Thirty-five (75\%) out of the 47 patients underwent surgery; among them, there were 15 patients with arterial thrombus, 2 patients with both venous and arterial thrombi, 12 patients with mesenteric/intestinal twist, 2 patients with

Table 2. Computed tomography findings in patients with mesenteric ischemia of different etiologies.

\begin{tabular}{|c|c|c|c|c|c|c|c|c|}
\hline Etiology & $\begin{array}{l}\text { Arterial } \\
\text { thrombus }\end{array}$ & $\begin{array}{l}\text { Venous } \\
\text { thrombus }\end{array}$ & $\begin{array}{l}\text { Bowel } \\
\text { thickening }\end{array}$ & $\begin{array}{l}\text { Bowel } \\
\text { distention }\end{array}$ & Ascites & $\begin{array}{c}\text { Portal } \\
\text { vein gas }\end{array}$ & $\begin{array}{l}\text { Pneuma- } \\
\text { tosis }\end{array}$ & Bowel enhancement \\
\hline $\begin{array}{l}\text { Arterial occlu- } \\
\text { sion }(n=19)\end{array}$ & $15(79 \%)$ & $2(11 \%)$ & $11(58 \%)$ & $13(68 \%)$ & $16(84 \%)$ & $5(26 \%)$ & $9(47 \%)$ & $\begin{array}{l}\text { Decreased }(\mathrm{n}=11,58 \%) \\
\text { Normal/increased }(\mathrm{n}=8,42 \%)\end{array}$ \\
\hline $\begin{array}{l}\text { Venous occlu- } \\
\text { sion }(n=13)\end{array}$ & $2(15 \%)$ & $10(77 \%)$ & $11(85 \%)$ & $5(38 \%)$ & $12(92 \%)$ & NONE & $5(38 \%)$ & $\begin{array}{l}\text { Decreased }(n=5,38 \%) \\
\text { Normal/increased }(n=8,62 \%)\end{array}$ \\
\hline $\begin{array}{l}\text { Mesenteric/intes- } \\
\text { tinal twist }(\mathrm{n}=12)\end{array}$ & NONE & NONE & $1(8 \%)$ & $12(100 \%)$ & $8(67 \%)$ & $2(17 \%)$ & $3(25 \%)$ & $\begin{array}{l}\text { Decreased }(n=8,67 \%) \\
\text { Normal/increased }(n=4,33 \%)\end{array}$ \\
\hline NOMI (n=3) & NONE & NONE & $3(100 \%)$ & $3(100 \%)$ & $3(100 \%)$ & $1(33 \%)$ & $3(100 \%)$ & $\begin{array}{l}\text { Decreased }(n=2,67 \%) \\
\text { Normal/increased }(n=1,33 \%)\end{array}$ \\
\hline
\end{tabular}


NOMI and remaining 4 patients were operated on based on decreased bowel enhancement on CT. The CT findings were concordant with surgical findings in $30(86 \%)$ patients. In the remaining $5(14 \%)$ cases, the additional findings were seen during surgery.

\section{Prognosis Based on the Time from Presentation to Imaging/Management}

Among 30 patients in Group A, 22 (73\%) individuals were operated on, and among them, the 2-week mortality was $90 \%(\mathrm{n}=20)$. The remaining 8 patients in Group A had venous thrombosis and were managed with anticoagulants with good outcomes. Among 17 patients in Group B, 13 (76\%) individuals were operated on, and the 2-week mortality was $62 \%(n=8)$. The remaining 4 patients included 1 individual with NOMI and 3 individuals with venous thrombosis who were managed conservatively with good outcomes.

\section{Discussion}

In our study, we evaluated 47 patients with clinical suspicion of mesenteric ischemia and a mean age of $57 \pm 11$ years the male-to-female ratio was $30(64 \%): 17$ (36\%). The clinical presentation included abdominal pain $(96 \%)$, nau- sea/vomiting (64\%), gastrointestinal bleeding (40\%) and signs of peritonitis $(21 \%)$. Serum lactate which has traditionally been used as a marker of mesenteric ischemia was elevated in $42 \%$ of our patients. These findings are quite similar to those of many previous studies, including those conducted by Corcos et al. [3], Clair et al. [11], Nuzzo et al. [13] and Gore et al. [14]. All these studies and the findings of our research point to the fact that clinical and laboratory features cannot be wholly relied upon when diagnosing or ruling out mesenteric ischemia. The symptoms in mesenteric ischemia are quite similar to many other acute abdominal conditions, ranging from inflammatory to infective and malignant. However, high mortality associated with mesenteric ischemia makes its early diagnosis essential for survival. Thus, there needs to be some diagnostic modality which is accurate enough to identify a patient with this condition.

An initial color Doppler USG was performed in all our patients. The common findings on USG were ascites, bowel thickening and distension. Bowel distension was common in cases of ischemia secondary to bowel/mesenteric twisting, leading to closed loop obstruction. We observed three patients with midgut volvulus. Five patients were found to have isolated spots of gas in the bowel wall, while 3 patients were believed to have PV gas. The identification of PV gas

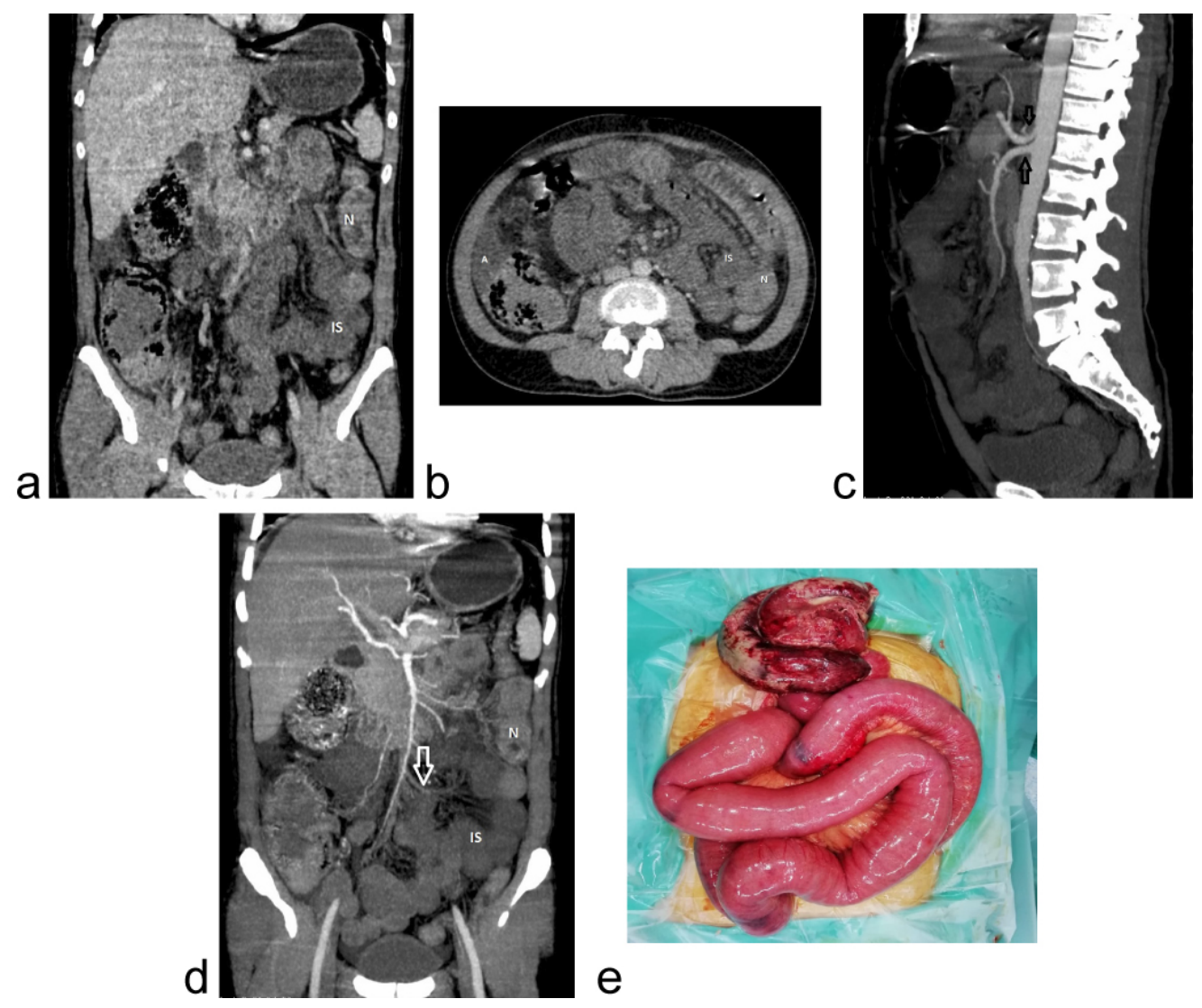

Figure 1. Mesenteric arterial thrombosis. Coronal (a) and axial (b) portal venous phase contrast-enhanced images taken from a 54-year-old male with abdominal pain showing hypoenhancing small bowel (IS) as compared to the normal gut (N). Sagittal CT angiography image (c) showing the normal origin and caliber of the superior mesenteric artery and the celiac axis (arrows). Coronal CT angiography image (d) showing the thrombus (arrow) in one of the branches of the superior mesenteric artery supplying the ischemic bowel (IS). Surgical image showing a portion of the non-viable bowel (dark colored) in comparison to normal pinkish bowel. This segment had to be resected. A - ascites. 


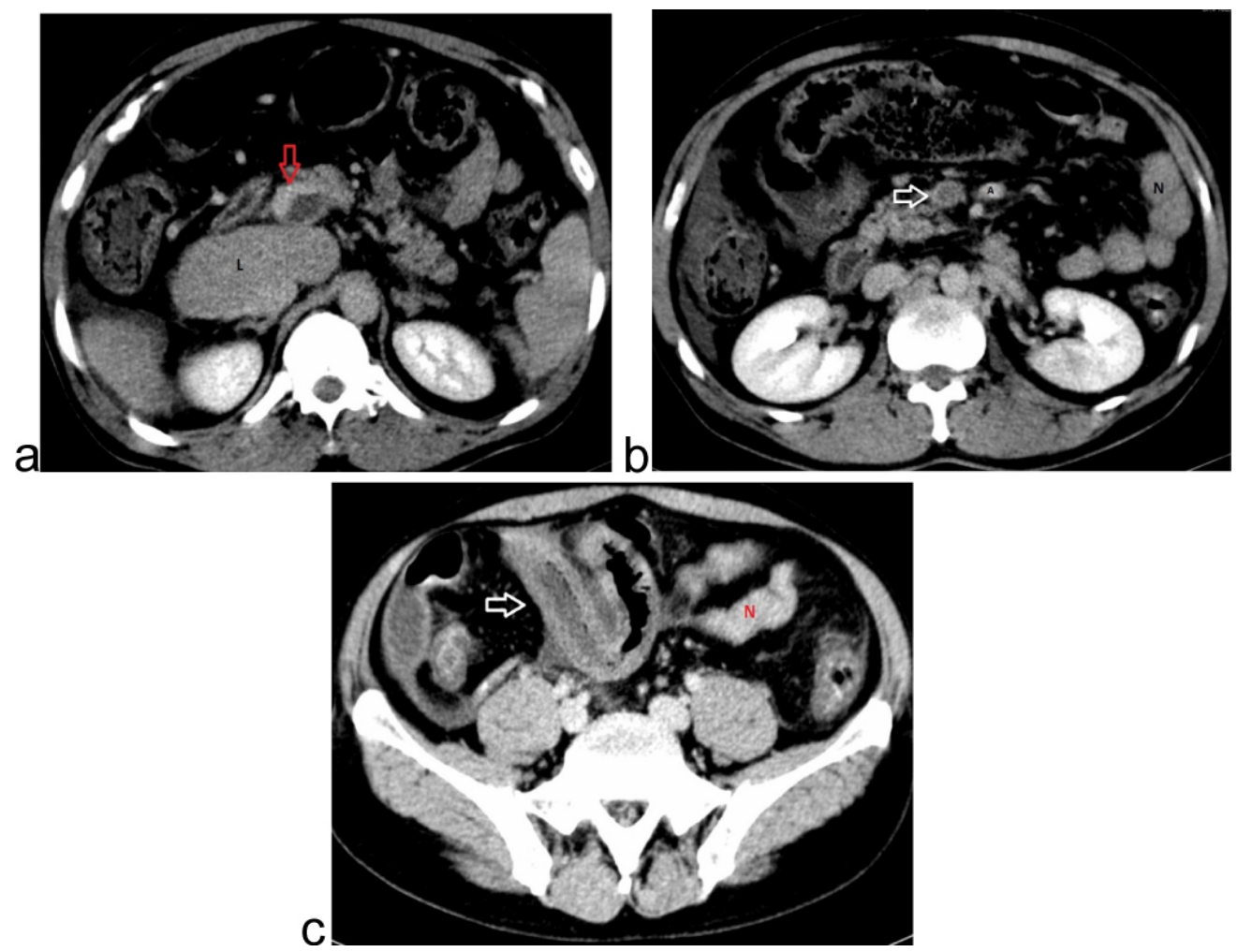

Figure 2. Mesenteric venous thrombus. Axial portal venous phase contrast-enhanced images (a, b) showing a partial thrombus in the portal vein (arrow - a) and complete thrombosis of the superior mesenteric artery (arrow - b). Axial contrast-enhanced CT image at a lower level showing a thickened but normally enhancing bowel. This patient was managed conservatively with anticoagulants and improved. $\mathrm{N}$ - normal bowel, A - the superior mesenteric artery.

on USG, especially its differentiation from pneumobilia, is difficult, therefore, caution should be exercised, especially in patients with no other signs of ischemia. The direct evidence of a vascular thrombus is quite difficult to pick up on USG; however, the addition of Doppler can help. We found direct evidence of arterial and venous thrombus in five and eight patients, respectively. This was, however, possible only in the proximal segments of the large vessels. The major disadvantage of USG is its operator dependence and requirement of good patient cooperation and preparation which are often lacking. Our findings are in concordance with studies evaluating the role of USG in mesenteric ischemia, including those conducted by Lassandro F et al. [15], Angelelli G et al. [16], Zwolak RM [17], Lewis BD \& James EM [18] and Haward TR et al. [19]. The conclusion about USG in mesenteric ischemia is that it is a useful initial screening imaging modality as it can rule out other non-urgent causes clinically mimicking mesenteric ischemia very well. Insofar as mesenteric ischemia is concerned, it can provide us with diagnostic clues, including bowel thickening, bowel distension and lack of peristalsis as indirect signs and arterial or venous thrombus visualization as direct signs of mesenteric ischemia, although the diagnostic accuracy is not high. In addition, USG lacks the ability to assess the mesenteric vasculature completely and no comment can be made about bowel wall vascularity or enhancement. Thus, USG remains a screening modality and should not be used to exclude a diagnosis of mesenteric ischemia, to say the least; however, it can add credence to the clinical and laboratory suspicion of mesenteric ischemia.

Contrast-enhanced computed tomography (CECT), including CT angiography, was performed in all patients to guide management. The direct evidence of arterial or venous thrombus was seen in $79 \%$ and $92 \%$ of patients, respectively (Fig. 1, 2).

CECT helped greatly in deciding the management of patients with mesenteric ischemia, arterial thrombosis and closed loop obstructions requiring surgical intervention or endovascular management, while patients with venous thrombosis were managed with anticoagulation. In 15 cases of arterial thrombosis, CT was able to identify the thrombus in the vessel and associated findings, including bowel thickening and distension, ascites and the hyperdense bowel contents. In 2 patients, there was noted an extension of aortic dissection into the mesenteric artery, leading to bowel ischemia (Fig. 3).

The patients with venous ischemia showed evidence of filling defects in the SMV, the portal and splenic veins associated with bowel thickening (water target sign), bowel distension, mesenteric congestion, and ascites (Fig. 2). Among patients with mesenteric/ intestinal twist, we found three cases of midgut volvulus and 9 cases of closed loop obstruction (3 internal hernias, 4 adhesive obstructions and 1 Meckel's diverticulitis associated with closed loop obstruction). In all these patients, CECT was able to detect the point of transition and decreased bowel enhancement along with dilated bowel loops (Fig. 4, 5). 

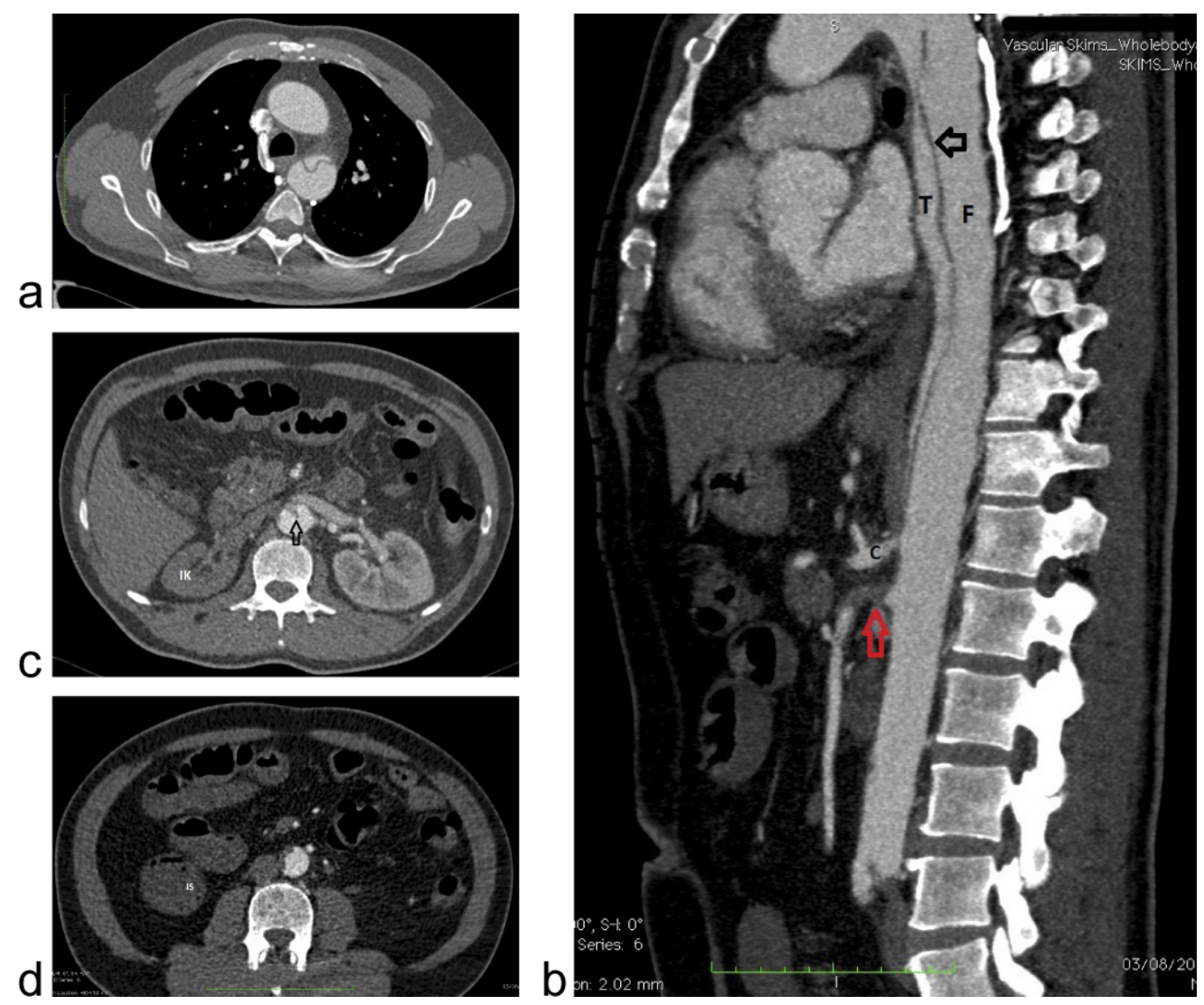

Figure 3. Type B aortic dissection with superior mesenteric artery extension. Axial (a) and sagittal (b) contrast-enhanced images showing the dissection flap (arrow) originating just below the origin of the left subclavian artery. True (T) and false $(F)$ lumens are indicated as well. The extension of the dissection flap into the superior mesenteric artery is indicated by the red arrow (b). Axial contrast-enhanced images (c, d) showing the effects of dissection in the form of the infracted right kidney (RK) and signs of early ischemic bowel changes (IS). This patient died on the surgical table.

We had 3 patients with NOMI, all three having chronic kidney disease and hypotension. The common findings in these patients were normal major mesenteric vessels, bowel thickening and distension, mesenteric congestion, pneumatosis intestinalis, and ascites (Fig. 6).

MPR contrast images can be used for assessing irregularities and spasm of the mesenteric arcade vessels which were seen in one of our patients. The diagnosis of NOMI can be challenging and angiography acts as the gold standard, but due to its invasive nature, it cannot always be used. Thus, in the underlying clinical conditions with CECT features described, a diagnosis of NOMI can be considered. Among the CT findings, the most ominous one was PV gas; all the patients $(n=8)$ with PV gas underwent bowel resection and none of them survived in the postoperative period (100\% mortality). The CECT findings described in our study are quite similar to many other studies [11, 2024]. Therefore, we are of the opinion that CECT with CT angiography should be performed in all patients with any suspicion of mesenteric ischemia on clinical, laboratory and USG findings. CECT helps in guiding management of these patients, as well as in identifying other abdominal conditions, mimicking mesenteric ischemia.

Surgery was performed in 35 patients and revealed arterial thrombus in 15 cases, both venous and arterial thrombi in 2 cases, mesenteric/intestinal twist in 12 cases and NOMI in 2 cases. The findings were somewhat concordant with the imaging findings. However, in 5 patients, who showed non-specific features of bowel thickening and distension and mesenteric congestion with ascites on CT, infracted bowel loops, requiring surgical resection were revealed. In addition, in two patients, CECT revealed nonenhancing bowel, and surgery revealed ischemic bowel that retained its viability after wrapping in warm saline pads. Among the patients undergoing surgery, bowel resection was required in $29(83 \%)$ cases, while $6(17 \%)$ patients responded to untwisting the mesentery/bowel, adhesiolysis, and warm saline flushes. Based on the correlation of CT findings with surgery, we have found that $\mathrm{CT}$ angiography has a sensitivity of $86 \%$, a specificity of $94 \%$ and an accuracy of $90 \%$. In 12 unoperated patients, a resolution of symptoms was observed during follow-up after treatment with anticoagulation, antibiotics and fluid therapy for hypotension, if necessary. These findings are quite similar to the study conducted by Zalcman et al. [25]. Therefore, barring a few limitations, CECT with angiography is a sine qua non as far as imaging mesenteric ischemia is concerned and guides management.

We observed a stark difference in the mortality rates of patients in Group A as compared to those in Group B. Those presenting and managed within 48 hours had an overall mortality of $63 \%$, while those presenting 48 hours after 
presentation had a mortality of $90 \%$. This difference was statistically significant ( $p<0.05$, Chi-Square test). This includes patients needing surgical intervention $(n=35)$. Those who were managed conservatively showed an overall good prognosis with only 2 mortalities attributed to other causes (1 patient with sepsis and the other patient with metabolic abnormalities leading to cardiac arrest). This has been previously illustrated by Ritz et al. [26]. We, therefore,
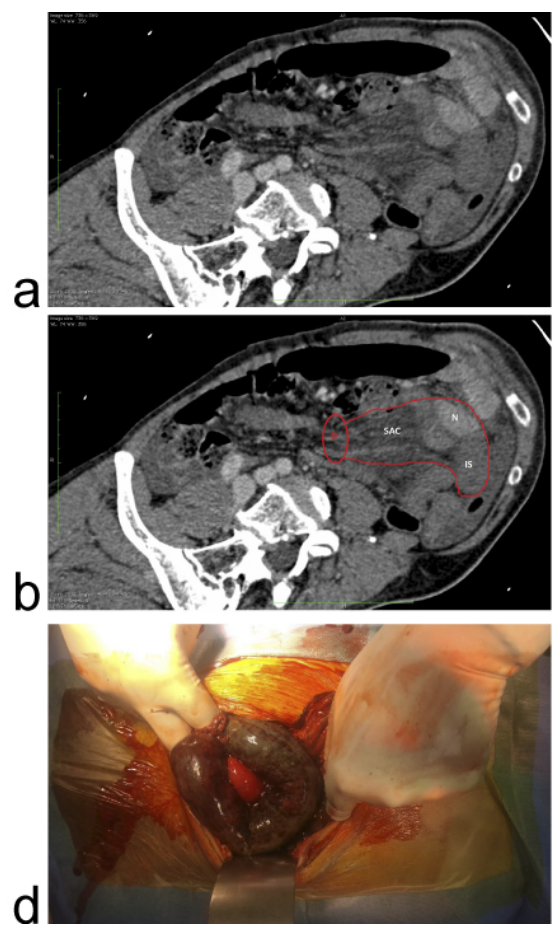

C

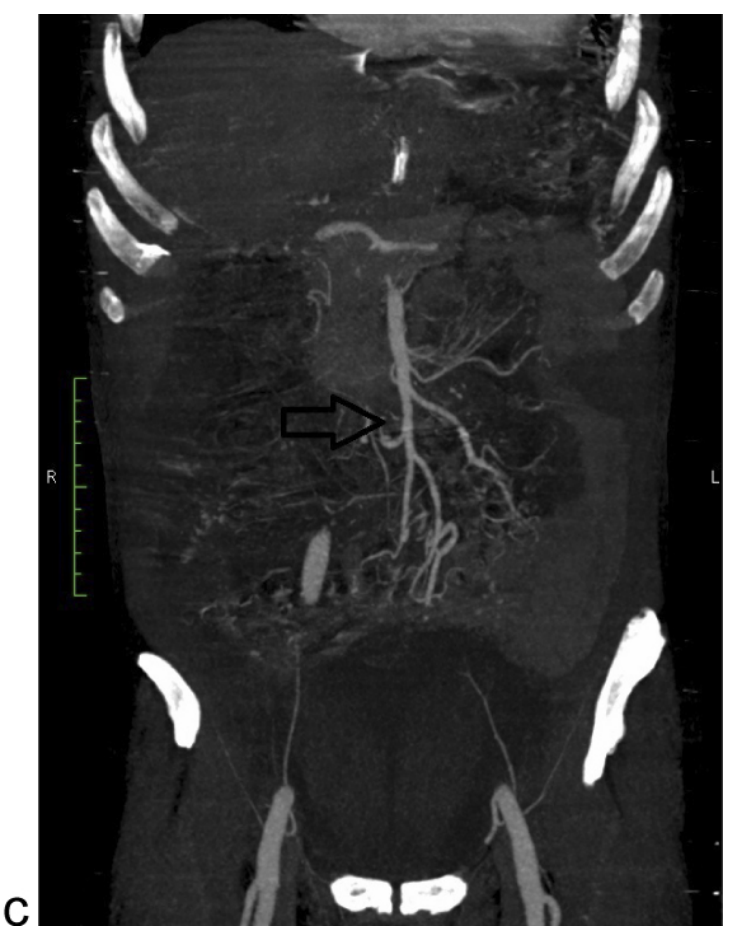

Figure 4. Internal hernia. Oblique axial portal phase contrast-enhanced multiplanar-reformation (MPR) images without (a) and with (b) annotations showing convergence of the mesenteric vessels to a point (red oval) corresponding to the mesenteric defect and the hernia sac is shown with both ischemic (IS) and normal (N) bowel loops. Coronal arterial phase contrast-enhanced MPR images showing normal mesenteric vasculature with no filling defects. Surgical image showing the gangrenous bowel and the adjacent normal bowel beneath it after retraction from the hernia sac. The defect (not seen directly) is indicated by the position of the right index finger.
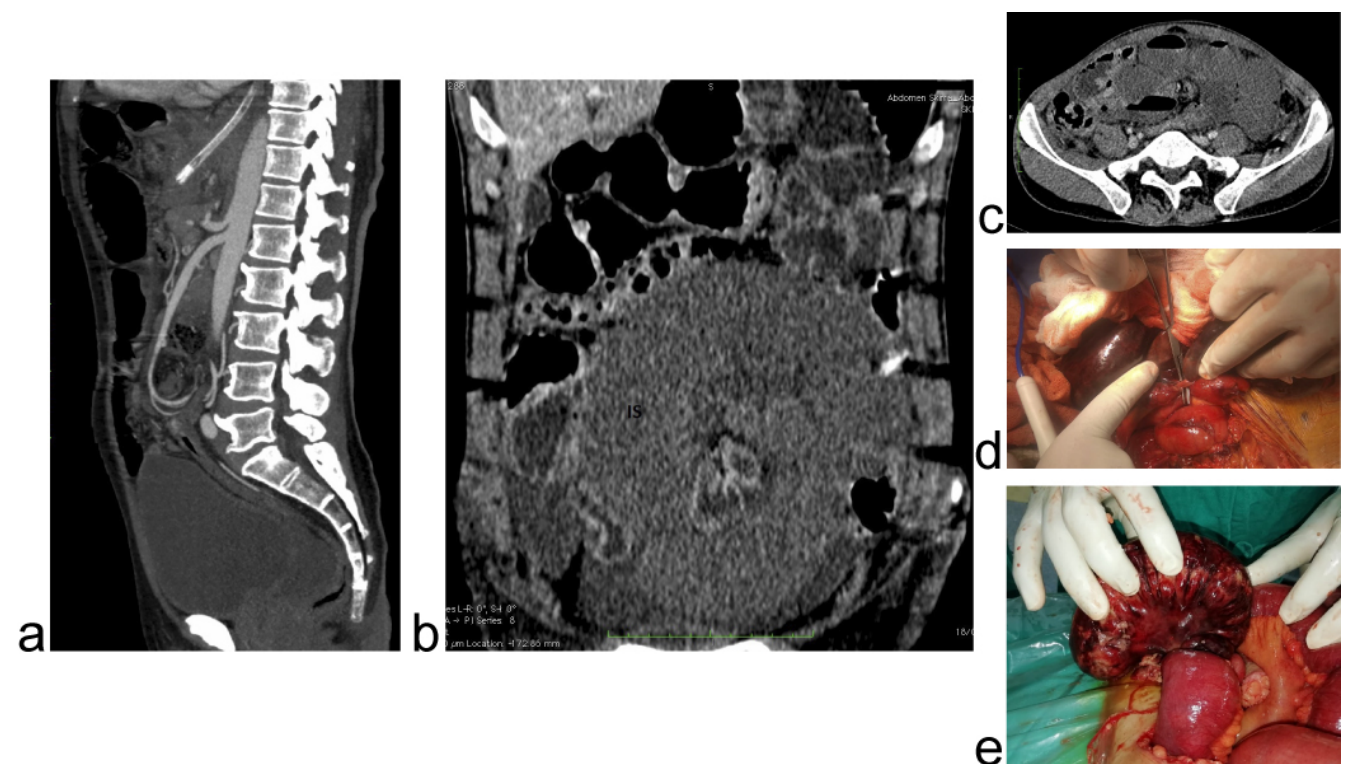

Figure 5. Closed loop obstruction. Sagittal (a) arterial phase contrast-enhanced MPR image showing normal mesenteric vasculature. Coronal (b) and axial (c) portal phase contrast-enhanced images showing dilated, non-enhancing bowel loop in $\mathrm{C} / \mathrm{U}$ configuration (IS). Axial images visualizing both abnormal (IS) and normal (N) bowel; an imaginary adhesive band (red curved line), which was the cause of obstruction (c). Surgical images (d, e) showing the presence of the adhesive band (d) with gangrenous bowel loops and the classical closed loop (e) with gangrenous changes. 

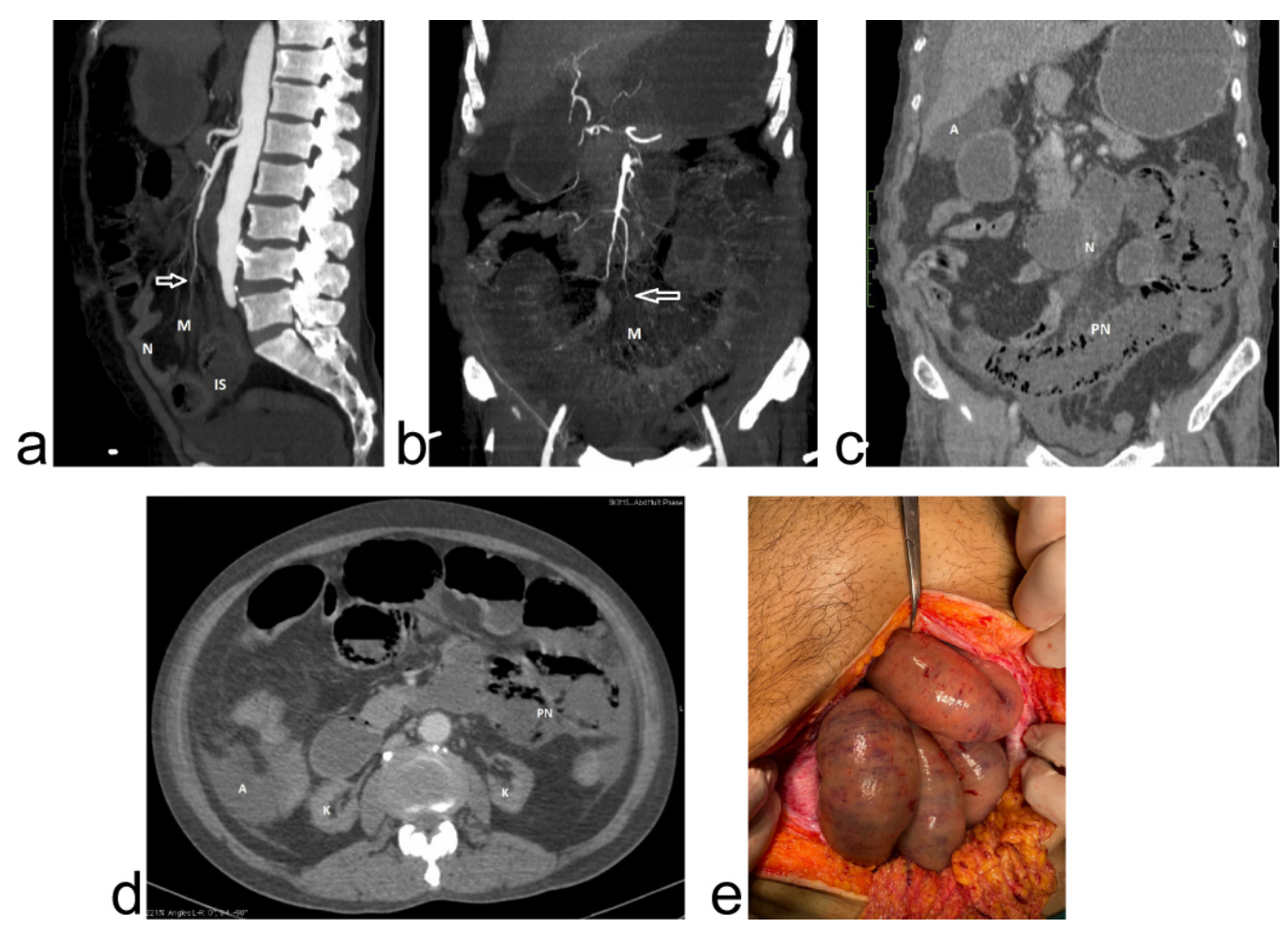

Figure 6. Non-occlusive mesenteric ischemia. Sagittal (a) and coronal (b) arterial phase contrast-enhanced maximum-intensity-projection (MIP) images taken from a 58-year-old male patient with chronic kidney disease showing multifocal narrowing (arrows) of the distal superior mesenteric artery branches and the mesenteric arcade with associated mesenteric congestion (M). Coronal (c) and axial (d) portal venous phase images showing abnormal bowel loops with pneumatosis $(\mathrm{PN})$, normal bowel loops $(\mathrm{N})$ and small atrophic kidneys $(\mathrm{K})$. Surgical image showing the presence of partially ischemic bowel which regained its viability after warm saline washes and no bowel resection was required. A ascites.

believe that, like other organs, the intestines might also have an ischemia time which, however, varies from patient to patient based on many physiological factors. However, a time of 48 hours can be considered as the golden time for patients with mesenteric ischemia beyond which mortality in these patients increases dramatically.

The limitations of our study include a relatively small size of the study population. A larger study with the assessment of mortality associated with the time of presentation is required to settle the golden period debate. The absence of endovascular management at our center is another limitation; however, such facilities are still not widely available in most parts of the developing world.

\section{Conclusions}

Clinical and laboratory features are non-specific and cannot be relied upon in patients with mesenteric ischemia. USG can be used as a screening modality in these patients. CT angiography can serve as a guiding light for management of patients with mesenteric ischemia. The presence of PV gas is an ominous sign with $100 \%$ mortality. A time of 48 hours from presentation can be considered as the golden period within which management of mesenteric ischemia can drastically reduce mortality.

\section{Ethical Statement \& Informed Consent}

As our study was an observational study with no clinical trial involved, the ethical clearance was not required; however, a written informed consent was taken from all the patients or their guardians with the surety that their names will be anonymised.

\section{Conflict of Interest}

The authors declare that no conflicts exist.

\section{Financial Disclosure}

The authors declared no funding or grant support.

\section{Acknowledgement}

Department of General and Minimal Invasive Surgery, SherI-Kashmir Institute of Medical Sciences, India.

\section{References}

[1] Mastoraki A. Mesenteric ischemia: pathogenesis and challenging diagnostic and therapeutic modalities. World Journal of Gastrointestinal Pathophysiology. 2016;7(1):125-130. Available from: https://doi.org/10.4291/wjgp.v7.i1.125 
[2] Herbert GS, Steele SR. Acute and chronic mesenteric ischemia. Surgical Clinics of North America. 2007;87(5):1115-1134. Available from: https://doi.org/10.1016/j.suc.2007.07.016

[3] Corcos O, Nuzzo A. Gastro-intestinal vascular emergencies. Best Practice \& Research Clinical Gastroenterology. 2013;27(5):709-725. Available from: https://doi.org/10.1016/j.bpg.2013.08.006

[4] Sardar P, White CJ. Chronic mesenteric ischemia: diagnosis and management. Progress in Cardiovascular Diseases. 2021;65:71-75. Available from: https://doi.org/10.1016/j.pcad.2021.03.002

[5] Huber TS, Björck M, Chandra A, Clouse WD, Dalsing MC, Oderich GS, et al. Chronic mesenteric ischemia: clinical practice guidelines from the Society for vascular surgery. Journal of Vascular Surgery. 2021;73(1):87S-115S. Available from: https://doi.org/10.1016/j.jvs.2020.10.029

[6] Danse EM, Kartheuser A, Paterson HM, Laterre PF. Color doppler sonography of small bowel wall changes in 21 consecutive cases of acute mesenteric ischemia. JBR-BTR. 2009;92(4):202-206.

[7] Menke J. Diagnostic accuracy of multidetector CT in acute mesenteric ischemia: systematic review and meta-analysis. Radiology. 2010;256(1):93-101. Available from: https://doi.org/10.1148/radiol.10091938

[8] Anglaret S, Dallongeville A, Beaussier H, Touloupas C, Boulay I, Tardivel AM, et al. Influence of clinical suspicion on CT accuracy of acute mesenteric ischemia: retrospective study of 362 patients. European Journal of Radiology. 2021;138:109652. Available from: https://doi.org/10.1016/j.ejrad.2021.109652

[9] Bala M, Kashuk J, Moore EE, Kluger Y, Biffl W, Gomes CA, et al. Acute mesenteric ischemia: guidelines of the World Society of Emergency Surgery. World Journal of Emergency Surgery. 2017;12(1):38. Available from: https://doi.org/10.1186/s13017-0170150-5

[10] Takiguchi T, Arai M, Kim S, Ishii H, Ogasawara T, Shigeta K, et al. Nonocclusive mesenteric ischemia associated with a hyperosmolar hyperglycemic state: hepatic portal venous gas as an indicator of mesenteric ischemia. Acute Medicine \& Surgery. 2021;8(1). Available from: https://doi.org/10.1002/ams2.673

[11] Clair DG, Beach JM. Mesenteric ischemia. New England Journal of Medicine. 2016;374(10):959-968. Available from: https://doi.org/10.1056/NEJMra1503884

[12] Bobadilla JL. Mesenteric ischemia. Surgical Clinics of North America. 2013;93(4):925-940. Available from: https://doi.org/10.1016/j.suc.2013.04.002

[13] Nuzzo A, Corcos O. Management of mesenteric ischemia in the era of intestinal stroke centers: the gut and lifesaving strategy. La Revue de Médecine Interne. 2017;38(9):592-602. Available from: https://doi.org/10.1016/j.revmed.2017.01.018

[14] Gore RM, Yaghmai V, Thakrar KH, Berlin JW, Mehta UK, Newmark GM, et al. Imaging in intestinal ischemic disorders. Radiologic Clinics of North America. 2008;46(5):845-875. Available from: https://doi.org/10.1016/j.rcl.2008.05.004

[15] Lassandro F, di Santo Stefano MLM, Maria Porto A, Grassi R, Scaglione M, Rotondo A. Intestinal pneumatosis in adults: diagnostic and prognostic value. Emergency Radiology. 2010;17(5):361-365. Available from: https://doi.org/10.1007/s10140-010-0868-9

[16] Angelelli G, Scardapane A, Memeo M, Stabile Ianora AA, Rotondo A. Acute bowel ischemia: CT findings. European Journal of Radiology. 2004;50(1):37-47. Available from: https://doi.org/10.1016/j.ejrad.2003.11.013

[17] Zwolak RM. Can duplex ultrasound replace arteriography in screening for mesenteric ischemia? Semin Vasc Surg. 1999;12:252-260.

[18] Lewis BD, James EM. Current applications of duplex and color doppler ultrasound imaging: abdomen. Mayo Clinic Proceedings. 1989;64(9):1158-1169. Available from: https://doi.org/10.1016/S00256196(12)64985-4

[19] Harward TRS, Smith S, Seeger JM. Detection of celiac axis and superior mesenteric artery occlusive disease with use of abdominal duplex scanning. Journal of Vascular Surgery. 1993;17(4):738-745. Available from: https://doi.org/10.1016/0741-5214(93)90119-7

[20] Oldenburg WA, Lau LL, Rodenberg TJ, Edmonds HJ, Burger CD. Acute mesenteric ischemia. Archives of Internal Medicine. 2004;164(10):1054-1062. Available from: https://doi.org/10.1001/archinte.164.10.1054

[21] Wasnik A, Kaza RK, Al-Hawary MM, Liu PS, Platt JF. Multidetector CT imaging in mesenteric ischemia-pearls and pitfalls. Emergency Radiology. 2010;18(2):145-156. Available from: https://doi.org/10.1007/s10140-010-0921-8

[22] Moschetta M. Multi-detector CT features of acute intestinal ischemia and their prognostic correlations. World Journal of Radiology. 2014;6(5):130-138. Available from: https://doi.org/10.4329/wjr.v6.i5.130

[23] Wiesner W, Khurana B, Ji H, Ros PR. CT of acute bowel ischemia. Radiology. 2003;226(3):635-650. Available from: https://doi.org/10.1148/radiol.2263011540

[24] Macari M, Balthazar EJ. CT of bowel wall thickening. American Journal of Roentgenology. 2001;176(5):1105-1116. Available from: https://doi.org/10.2214/ajr.176.5.1761105 
[25] Zalcman M, Sy M, Donckier V, Closset J, Gansbeke DV. Helical CT signs in the diagnosis of intestinal ischemia in small-bowel obstruction. American Journal of Roentgenology. 2000;175(6):1601-1607. Available from: https://doi.org/10.2214/ajr.175.6.1751601

[26] Ritz J-P, Germer C-T, Buhr HJ. Prognostic factors for mesenteric infarction: multivariate analysis of 187 patients with regard to patient age. Annals of Vascular Surgery. 2005;19(3):328-334. Available from: https://doi.org/10.1007/s10016-005-0005-5

Received: 2021-10-17

Revision Requested: 2021-11-15

Revision Received: 2021-11-21

Accepted: 2021-11-28 